\title{
28 Research Square

\section{Imaging the Alternatively Spliced D Domain of Tenascin C in Preclinical Models of Inflammatory Bowel Disease}

Liang Zhang ( $\sim$ liang.zhang@abbvie.com )

AbbVie Bioresearch Center https://orcid.org/0000-0001-5766-2302

Calvin S. Pohl

AbbVie Bioresearch Center

Kristoff T. Homan

Bristol Myers Squibb

Victor Z. Sun

AbbVie Bioresearch Center

Stephanie M. Gaudette

Worcester Technical High School

Christine M. Nelson

AbbVie Bioresearch Center

Jamie E. Erickson

AbbVie Bioresearch Center

Heather L. Knight

AbbVie Bioresearch Center

Sahana Bose

AbbVie Bioresearch Center

Grace R. Lynch

AbbVie Bioresearch Center

Bradford L. McRae

AbbVie Bioresearch Center

Annette J. Schwartz Sterman

AbbVie Bioresearch Center

Soumya Mitra

AbbVie Bioresearch Center

\section{Research Article}

Keywords: Alternatively Spliced D Domain, Tenascin, Preclinical Models, Inflammatory Bowel Disease, mouse colitis models 
Posted Date: October 20th, 2021

DOl: https://doi.org/10.21203/rs.3.rs-984872/v1

License: (c) (i) This work is licensed under a Creative Commons Attribution 4.0 International License. Read Full License 


\section{Abstract}

Purpose To image colon-expressed alternatively spliced D domain of tenascin C in preclinical colitis models using near infrared (NIR) labeled targeted molecular imaging agents.

Methods Human IgG and ScFv fusion proteins specific to the alternatively spliced D domain of tenascin C were generated. Immunohistochemistry identified disease-specific expression of this extracellular matrix in mouse colitis models. Proteins were labeled with the NIR fluorophore IRDye $800 \mathrm{CW}$ via amine chemistry and intravenously dosed to evaluate targeting specificity in preclinical rodent and primate colitis models.

Results The NIR labeled proteins successfully targeted colonic lesions in a murine model of colitis and appeared as distinct punctate spots. Co-administration of a blocking dose reduced the whole colon standardized uptake of the fluorescent dose $>7$-fold in mouse models. Estimates suggest local expression at $>100 \mathrm{nM}$ in diseased mouse colon. Macroscopic targeting specificity was not observed in diseased primate colon. Cellular level specificity was assessed via microscopy and immunohistochemistry.

Conclusion Our imaging data suggest the alternatively spliced D domain of tenascin $\mathrm{C}$ is a promising target for delivery-based applications in inflammatory bowel diseases.

\section{Introduction}

Inflammatory bowel diseases (IBD) are progressive disorders of the gastrointestinal tract that hinder the patient's quality of life if left untreated. Common intervention therapies include anti-inflammatory and immunosuppressive agents such as 5 -amino salicylates for mild cases and corticosteroids in more severe cases [1, 2]. More recently, in light of the evidence for the crucial roles of cytokine production and signaling in IBD pathogenesis, biologic therapeutics have emerged with the potential to induce remission and heal the intestinal mucosa [2-4]. Unlike small molecule approaches, which can be formulated for tailored release via oral administration [5], larger molecular weight biologics often rely on parenteral dosing. These systemic treatments have yielded mixed results in mouse models and the clinic, where a lack of selective targeting and delivery has yielded low drug concentrations in the colon while accompanying unwanted increases in serum acute-phase proteins [6-8].

Extracellular matrix (ECM) proteins are upregulated in a variety of inflammatory diseases due to increased tissue remodeling and have served as attractive imaging and drug delivery targets [9-12]. Increased ECM expression is well-documented in IBD, where remodeling is a consistent feature implicated in disease pathogenesis [13], and elevated serum levels of ECM proteins such as tenascin C are observed in IBD patients [14]. Tenascin $\mathrm{C}$ is a glycoprotein with multiple functional isoforms within the ECM. While some isoforms are expressed in healthy tissue, others such as isoforms containing domain D and A1 have been used in the clinic for delivery of radiolabeled antibodies due to their selective expression in tumor tissue [15-17]. Additionally, single chain Fv (scFv) targeting tenascin C have been shown to 
specifically accumulate in tumors in several oncology models [18]. A related ECM target for deliverybased strategies, fibronectin extra-domain $\mathrm{A}$, has seen promising transition from oncology to IBD and is well-reported [19]. However, tenascin C isoforms remain largely unexplored in IBD models, despite its reported expression. For example, in a commonly used murine colitis model, the addition of dextran sulfate sodium (DSS) to drinking water resulted in tenascin $C$ upregulation in damaged mucosal areas [20]. Additionally, Kawamura et al. demonstrated a marked increase of both colonic protein and gene expression of tenascin $\mathrm{C}$ in a murine model of colitis associated cancer [21]. Perhaps most notable, similar to the identification of fibronectin extra-domain $\mathrm{A}$, in vivo protein biotinylation followed by liquid chromatography-tandem mass spectrometry also identified the alternatively spliced $D$ domain of tenascin C (herein referred to as TNC D) in diseased mouse colon extracts [22-24]. Here, targeted molecular imaging can answer key questions involving disease expression, target engagement, and feasibility of locally delivered therapeutics [25-27].

Near infrared (NIR) imaging is increasingly used to elucidate the tissue level distribution of various clinically relevant antibodies [28-31]. Not only is reagent generation time- and cost-effective, the lack of ionizing radiation and increased resolution make it an attractive modality for preclinical target identification. Despite these benefits, the overlap between NIR imaging and therapeutics development to evaluate novel antigens for targeted drug delivery is less common in IBD research. Instead, common colon therapeutic delivery methods include orally dosed nanoparticle formulations that rely on increased intestinal permeability or pharmacologically inactive prodrugs that require enzymatic catalysis [5, 32]. Due to incompatible labeling chemistries and risks of altering bioavailability-owing to the physicochemical properties of the detectable label-these delivery platforms often preclude molecular imaging for target engagement. Several positron emission tomography (PET) probes such as $\left[{ }^{18} \mathrm{~F}\right] \mathrm{FDG}$ for glucose metabolism and ${ }^{89} \mathrm{Zr}$-labeled cys-diabodies for $\mathrm{CD} 4+\mathrm{T}$ cells have seen use in IBD research [33-36]. However, these probes focus on inflammation imaging and the target expression is often not unique to colon. Thus, increasing the repertoire of antigens unique to diseased colon is fruitful and molecular imaging can help identify and verify high expressing antigens. In this study, we generated novel antibody-based reagents specific to TNC D. Using immunohistochemistry (IHC) expression in clinical and preclinical colon samples as a guide, we evaluated TNC D as an in vivo target in preclinical IBD models using NIR imaging.

\section{Methods}

\section{Expression and purification}

ABBV-022 was transiently expressed in $\mathrm{CHO} 3 \mathrm{E} 7$ cells for 7 days following transfection. Anti-TNC D IgG production is similar to previously described $[10,18]$. The monoclonal antibody targeting TNC $D$ and ABBV-022 were purified via protein A through mediated capture of the Fc and VH3 domains of the IgG and SCFv, respectively. For each TNC D targeting protein, the clarified supernatant was purified on a AKTAPure FPLC (Cytiva, Marlborough, MA) via ProteinA affinity capture using a HiTrap MabSelect SuRe 
column (Cytiva, Marlborough, MA) and eluted at low pH. A second purification was performed using a Superdex 200 pg (Cytiva, Marlborough, MA) size exclusion column using tris buffered saline to yield $>95 \%$ protein purity as determined by analytical liquid chromatography (Agilent, Santa Clara, CA). Endotoxin level were quantified to be $<1 \mathrm{EU} / \mathrm{mg}$ (Charles River Laboratories, Wilmington, MA).

\section{Binding assays}

Binding kinetics for $800 \mathrm{CW}$ labeled and unlabeled anti-TNC D IgG and ABBV-022 against recombinant soluble TNC were measured via surface plasmon resonance on a BIAcore T200 instrument (GE Healthcare, Uppsala, Sweden) at $25^{\circ} \mathrm{C}$. Measurements for TNC D IgG were performed using Fc capture, whereas ABBV-022 was directly immobilized on chip. Either $10000 \mathrm{RU}$ of goat anti-human Fc polyclonal antibody (Thermo Fisher Scientific Inc., \#31170) or 2000 RU of ABBV-022 was diluted to $20 \mu \mathrm{g} / \mathrm{mL}$ or 2 $\mu \mathrm{g} / \mathrm{mL}$, respectively, in $10 \mathrm{mM}$ sodium acetate $(\mathrm{pH} 4.5)$ and was immobilized on all four flow cells across CM5 chip via amine coupling followed by $1 \mathrm{M}$ ethanolamine quench. Flow cell 1 was used as a reference. Chip preparation and binding kinetic measurements were made in the assay buffer HBS-EP+ $(10 \mathrm{mM}$ HEPES, pH 7.4, $150 \mathrm{mM} \mathrm{NaCl}, 3 \mathrm{mM}$ EDTA, 0.05\% Tween 20). For the IgG, assay cycles consisted of capture of the anti-TNC D IgG molecule at $1 \mu \mathrm{g} / \mathrm{mL}$ and a flow rate of $5 \mu \mathrm{L} / \mathrm{min}$ for $60 \mathrm{~s}$ followed by analyte injection of either murine tenascin or buffer control over both reference and active surface for 300 $s$ at $50 \mu \mathrm{L} / \mathrm{min}$, after which the dissociation was monitored for $300 \mathrm{~s}$ at $50 \mu \mathrm{L} / \mathrm{min}$. For ABBV-022, cycles consisted of either murine tenascin or buffer control over both reference and active surface for $300 \mathrm{~s}$ at $50 \mu \mathrm{L} / \mathrm{min}$, after which the dissociation was monitored for $300 \mathrm{~s}$ at $50 \mu \mathrm{L} / \mathrm{min}$. Tenascin injections were flowed at increasing concentrations from $1.8 \mathrm{nM}$ to $1000 \mathrm{nM}$. Data were processed and fitted globally to a 1:1 binding model using BIAcore T200 evaluation software to determine the binding constants.

\section{Immunohistochemistry}

Colons from C57BL/ 6 mice on 3\% DSS in the drinking water for 7 days were either embedded in OCT or fixed in $4 \%$ paraformaldehyde (PFA) and sucrose-protected. OCT-embedded sections were acetone-fixed same day as sectioning and air-dried overnight. Both acetone-fixed and PFA/sucrose-protected $5 \mu \mathrm{m}$ frozen sections were brought to room temperature prior to IHC. Tissue sections were blocked with both dual endogenous block and protein block (both Agilent) prior to a 30 min incubation with either anti-TNC D or isotype $\mathrm{IgG}$ at $0.03 \mu \mathrm{g} / \mathrm{ml}$. The primary human antibodies were detected sequentially with rabbit antihuman IgG (Southern Biotech, Birmingham, AL), HRP polymer, and visualized with DAB with a hematoxylin counterstain (Leica Bond Polymer Refine Detection kit). Following immunolabeling and detection, the glass slides were imaged using a Pannoramic 250 whole slide scanner at 20x magnification (3D Histech, Budapest, Hungary). To visualize the NIR signal at a microscopic scale, $10 \mu \mathrm{m}$ sections colon samples were counterstained with Hoechst 33342 (Invitrogen, Waltham, MA) and imaged using a Pannoramic 250 slide scanner at 20x magnification.

Clinical IBD and diseased cynomolgus macaque colon were evaluated using a biotinylated anti-TNC D antibody. Frozen human tissue was obtained from CHTN (National Cancer Institute, Bethesda, MD), Folio Biosciences (Columbus, OH), and UMASS Memorial Gastroenterology (Worcester, MA). Frozen human 
sections were post-fixed with acetone on the day of sectioning and air-dried overnight. Frozen cynomolgus tissue was fixed in 4\% PFA and sucrose-protected. Both acetone-fixed and PFA/sucroseprotected $5 \mu \mathrm{m}$ frozen sections were brought to room temperature prior to IHC. Tissue sections were blocked with dual endogenous block, a streptavidin/biotin blocking kit (Vector Labs, Burlingame, CA), and a protein block, followed by a 15 min incubation with human anti-TNC D IgG at $0.7 \mu \mathrm{g} / \mathrm{ml}$. The biotinlabeled, anti-TNC D IgG was detected using Vectastain Elite ABC-HRP reagent (Vector Labs, Burlingame, $\mathrm{CA}$ ) and visualized with DAB with a hematoxylin counterstain (Leica Bond Polymer Refine Detection kit). Slides were scanned on a Pannoramic 250 slide scanner.

\section{IRDye 800CW conjugation}

Solutions of ABBV-022, anti-TNC D IgG, or isotype lgG were buffered exchanged to PBS using $7 \mathrm{kDa}$ MWCO ZEBA desalting columns. To these protein solutions ( $50 \mathrm{nmol}$ in $1 \mathrm{~mL}$ ), $100 \mu \mathrm{L}$ of $7.5 \%$ sodium bicarbonate was added. The mixture was mixed by inverting before addition of IRDye 800CW NHS ester (40 nmol in DMSO). The solution was mixed again by gently inverting and reacted at room temperature for $2 \mathrm{~h}$. Unreacted and hydrolyzed $800 \mathrm{CW}$ dye was removed using two Zeba desalting columns (7 kDa MWCO). Fluorophore degree of label was determined using a NanoDrop 2000c spectrophotometer (ThermoFisher Scientific, Waltham, MA). Monomer purity was determined via SEC-HPLC with $280 \mathrm{~nm}$ and $770 \mathrm{~nm}$ detection.

\section{Animals}

Mice studies were conducted at AbbVie to the standards of the Association for the Assessment and Accreditation of Laboratory Animal Care (AAALAC) standards. All studies with mice were performed according to approved protocols by AbbVie's Institutional Animal Care and Use Committee (IACUC). Briefly, seven-day acute murine colitis was induced in wild-type C57BL/6 mice with $3 \%$ DSS (MP Biomedicals, Santa Ana, CA) in the drinking water. On day 7, animals were intravenously dosed with NIRlabeled reagents for imaging. Mice body weights were monitored, and animals were euthanized if $>20 \%$ of the initial weight was lost. All nonhuman primates were female Chinese cynomolgus macaques, between 3-5 years old, and were acquired from Charles River Laboratories. All primates tested negative for viral and enteric pathogens and tuberculosis prior to initiation of the study. Primates were house at AbbVie Inc, in Lake County, IL, in compliance with AAALAC standards, and all procedures were reviewed and approved by the AbbVie Inc, IACUC (Protocol ID: 1705C00021). Primate experimental chemical colitis was established with trinitrobenzene sulfonic acid (TNBS). TNBS (Sigma-Aldrich, St. Louis, MO) was administered in $1 \mathrm{~mL}$ volume of $50 \%(\mathrm{v} / \mathrm{v})$ ethanol. Day 0 application of TNBS was at $100 \mathrm{mg}(10 \% \mathrm{w} / \mathrm{v}$ solution) with subsequent applications on day 7 and day 14 at $50 \mathrm{mg} \mathrm{(5 \%} \mathrm{w/v} \mathrm{solution).} \mathrm{Applications}$ were administered intrarectally (IR) with endoscopic guidance to distances of $15-18 \mathrm{~cm}$ in the colon, and primates incubated in dorsal recumbency with their posterior elevated for $10 \mathrm{~min}$. The primates were euthanized with Fatal-Plus (Vortech Pharmaceuticals, Dearborn, MI) $48 \mathrm{~h}$ post imaging agent dose.

\section{Plasma clearance}


To determine the blood clearance rates for the ABBV-022-800CW, anti-TNC D IgG-800CW, and isotype IgG$800 \mathrm{CW}$ conjugates, C57BL/ 6 mice were intravenously dosed with $0.7 \mathrm{nmol}$ of NIR labeled protein via the tail vein on day 7 of DSS. At predetermined timepoints, $20 \mu \mathrm{L}$ of whole blood was collected via a tail nick and mixed with $80 \mu \mathrm{L}$ of $10 \mathrm{mM}$ EDTA in PBS. The blood samples were mixed, and frozen at $-80^{\circ} \mathrm{C}$. The samples were later thawed and $800 \mathrm{CW}$ fluorescence was measured using an Odyssey CLx (Li-Cor, Lincoln, NE). For the primates, ABBV-022-800CW was administered intravenously at $2 \mathrm{mg} / \mathrm{kg}$, three days after the final dose of TNBS (day 17), and whole blood was collected from conscious, chaired cynomolgus macaques at $10 \mathrm{~min}, 30 \mathrm{~min}, 1 \mathrm{~h}, 3 \mathrm{~h}, 6 \mathrm{~h}, 24 \mathrm{~h}$, and $48 \mathrm{~h}$ post dose. Fluorescence was converted to whole blood concentration using a standard curve to quantify the absolute concentration of fluorescent protein in the blood similar to previously published methods [37]. The clearance was fit to a biexponential decay. Fitted initial concentration were compared with theoretical values based on dose and total blood volume.

\section{Ex vivo biodistribution}

Following the last blood draw, mice were euthanized and organs were resected and imaged on a Pearl Imager (Li-Cor, Lincoln, NE). To quantify \%ID/g, a previously published protocol was adopted [38]. Briefly, organs were weighed, and incubated at $37^{\circ} \mathrm{C}$ in a mixture of RIPA buffer in PBS supplemented with 6 $\mathrm{mg} / \mathrm{ml}$ collagenase type IV for $30 \mathrm{~min}$. An equal volume of $0.25 \%$ trypsin/EDTA was added and the solution was incubated for another 30 min before being homogenized using a FB-120 Sonic

Dismembrator. Known amounts of digested NIR conjugates were used to generate a calibration curve and bulk signal from organ digests was converted to absolute concentrations to quantify the \%ID/g. For primates, $48 \mathrm{~h}$ after dose, the animals were euthanized and distal colons were resected. Diseased and healthy segments of the distal colon from each animal were identified by eye and imaged with a Pearl Imager.

\section{Near infrared fluorescent molecular endoscopy}

Female $\mathrm{C} 57 \mathrm{BI} / 6$ mice were provided DSS in drinking water as previously described for disease induction. On day 8 , animals were intravenously dosed with $0.7 \mathrm{nmol}$ of ABBV-022-800CW. One day later, animals were placed under anesthesia with isoflurane. The colon was flushed with PBS by gently inserting a flexible gavage needle $3 \mathrm{~cm}$ into the anus to remove feces. Animals were removed from anesthesia and allowed to ambulate in order to pass any fecal pellets that remained. The animals were again placed back under anesthesia and a Karl Storz sheath was used to insert a SurgVision Explorer endoscope (Munich, Germany) in the anus. Images of the colon were captured from $3 \mathrm{~cm}$ to $0.5 \mathrm{~cm}$ from the anal verge; single frame images were extracted from the time lapse using SurgVision software.

\section{Results}

Surface representations for anti-TNC D IgG and ABBV-022 are shown (Fig. 1A). ABBV-022 consists of full length human IL-22 protein fused to the N-terminus of TNC D targeting ScFv via a 15 amino acid linker. The $\mathrm{VH}$ and $\mathrm{VL}$ domains of scFv are linked by a short flexible 5 amino acid linker (GGSGG) to prevent 
intrachain folding and drive stability. Thus, the resulting diabody is formed as a noncovalent dimer of two IL-22-scFv molecules coming together in solution stabilized by interchain VH/VL interactions. Sequence alignment of TNC D from human, rat, cynomolgus monkey, and mice show conservation across human and model organisms (Fig. 1B). No significant differences were observed in either on-rate or off-rate kinetics between labeled and unlabeled proteins (Table 1). Near 2.5-fold difference was observed in the on-rate between the IgG and ABBV-022 (1.4 x $10^{5}$ vs $\left.5.5 \times 10^{4} \mathrm{M}^{-1} \mathrm{~s}^{-1}\right)$. TNC D is expressed in the lamina propria of patients with Crohn's disease and ulcerative colitis (Fig. 2). In the preclinical mouse colitis model, staining is observed at the site of lesions throughout the lamina propria down to the muscularis mucosae. This staining pattern is not observed in healthy samples, where TNC D can be found in the superficial lamina propria, in proximity to mucosal epithelial cells.

Anti-TNC D IgG, ABBV-022, and isotype IgG were labeled with the NIR fluorophore IRDye 800CW to evaluate in vivo tenascin targeting in a murine DSS colitis model. Systemic clearance of the NIR labeled proteins following a $0.7 \mathrm{nmol}$ bolus intravenous dose indicated faster clearance phase kinetics for the ABBV-022-800CW compared to the fluorescent IgG groups $\left(t_{1 / 2, b}=19 \pm 3 \mathrm{~h}, 20 \pm 1 \mathrm{~h}\right.$, and $5.2 \pm 0.3 \mathrm{~h}$, for the anti-TNC D IgG, isotype IgG, and scFv fusion, respectively), consistent with a lack of FcRn recycling (Fig. 3A). Biexponential fit parameters suggest a more rapid distribution phase for ABBV-022-800CW $\left(\mathrm{t}_{1 / 2, \mathrm{a}}=0.5 \pm 0.2 \mathrm{~h}\right)$, in agreement with its lower molecular weight (Table 2). Co-dosing $20 \mathrm{nmol}$ of unlabeled anti-TNC D IgG minimally impacted the pharmacokinetics of the anti-TNC D IgG-800CW $\left(\mathrm{t}_{1 / 2, \mathrm{~b}}=\right.$ $21 \pm 3 \mathrm{~h}, 19 \pm 3 \mathrm{~h}$ for with and without blocking doses, respectively, $\mathrm{P}=0.5)$. Curiously, the isotype IgG$800 \mathrm{CW}$ also exhibited rapid distribution phase kinetics; however, at the time of imaging, systemic concentrations for the isotype matched that of anti-TNC D groups. Animals were euthanized $24 \mathrm{~h}$ and 48 $\mathrm{h}$ following dosing for ABBV-022-800CW and IgG groups, respectively. Colons were resected, flushed, and imaged (Fig. 3B). Macroscopic images revealed punctate lesions spanning the distal and proximal colon in animals dosed with anti-TNC D IgG-800CW. Fluorescent intensity was reduced in the ABBV-022-800CW dosed group, likely due to a combination of faster clearance and reduced binding affinity compared to the IgG. Co-dosing $20 \mathrm{nmol}$ of unlabeled anti-TNC D IgG with $0.7 \mathrm{nmol}$ anti-TNC D IgG-800CW reduced the lesional fluorescence to isotype IgG-800CW levels. Co-dosing $20 \mathrm{nmol}$ of unlabeled anti-TNC D IgG with $0.7 \mathrm{nmol}$ ABBV-022-800CW similarly reduced macroscopic punctate signal (Fig. S5).

For the ex vivo biodistribution, organs were weighed, homogenized, and imaged with digest standards to quantify normalized uptake (Fig. 4A). In agreement with the imaging results, blocking with $20 \mathrm{nmol}$ of unlabeled anti-TNC D IgG reduced total colon uptake for the targeting constructs $(1.5 \pm 0.2$ vs $0.20 \pm 0.04$ $\% \mathrm{ID} / \mathrm{g}$ for unblocked and blocked for $\lg \mathrm{G}$, respectively, $\mathrm{P}=0.001 ; 0.60 \pm 0.04 \mathrm{vs} 0.2 \pm 0.1 \% \mathrm{ID} / \mathrm{g}$ for unblocked and blocked ABBV-022-800, respectively, $\mathrm{P}=0.03$ ). Higher ABBV-022-800CW homogenate signal was also observed in liver compared to the IgG format. To explore if these differences were due to the fate of the fluorescent label, rather than in vivo targeting, ${ }^{125}$-substitution was performed with the scFv and organ uptake was compared to the NIR data (Fig. S6). While small differences in colon uptake were observed between ABBV-022-800CW and [ $\left.{ }^{125}\right]$ ABBV-022, there was a marked reduction in liver uptake for the iodinated compound (Fig. S7). This is expected as iodine-125 can be trafficked out of cells 
via diffusion or amino acid transporters once the parental protein is degraded. On the other hand, IRDye $800 \mathrm{CW}$ is a residualizing label that slowly diffuses out of cells once internalized [39]. Thus, the measured NIR signal is more reflective of protein metabolism in the liver, rather than liver targeting. A blocking dose of anti-TNC D IgG also did not reduce NIR signal in the liver, suggesting a lack of specific TNC $D$ binding ( $5.5 \pm 1.1$ vs $5.3 \pm 4.7 \% \mathrm{ID} / \mathrm{g}$ for unblocked and blocked for lgG, respectively, $\mathrm{P}=0.9$ ). No significant differences in uptake were observed in spleen, lung, and muscle among all dosed groups (Fig. 4A). With clinical relevance in mind, video data were recorded using a similar and previously reported fluorescent molecular endoscope equipped with a NIR excitation source [40,41]. Here, punctate fluorescent regions consistent with lesion-associated TNC D expression were observed and agreed well with ex vivo images (Fig. 4B). Due to similarities between this platform and the clinically adopted instrument, the preclinical data highlight possible avenues for imaging such as tracking drug distribution or diagnostics. Lastly, we sought to confirm cellular level specificity and in vivo stability for the TNC D targeting proteins. Strong NIR fluorescence is observed in regions of focal erosion in the lamina propria as confirmed through sequential histology sections (Fig. 4C). A magnified image shows agreement with tenascin IHC (Fig. 2). Consistent distribution between the NIR signal and IHC using an anti-human IgG suggests minimal metabolism and trafficking of the NIR label in diseased colon (Fig. S8).

ABBV-022-800CW was intravenously dosed in a cynomolgus macaque model of TNBS colitis. Segments of disease and healthy colon from the same animal were resected and imaged, and the NIR mean fluorescence intensity (MFI) for these colon segments were plotted (Fig. 5A). Colon segment images showed a diffuse pattern compared to mouse and a greater than 2-fold increase in NIR MFI was observed in diseased colon segments compared to healthy colon segments $(0.34 \pm 0.05$ vs $0.12 \pm 0.08$ for diseased vs healthy, respectively, $P=0.03$ ). Similar to ABBV-022-800CW, a 2-fold increase in NIR MFI was observed diseased vs healthy colon segments with a non-targeting ScFv control. Disease site punch biopsies identified by eye were also collected and homogenized to estimate the local protein concentration (Fig. 5B). The fold changes in concentration between ABBV-022-800CW and the nontargeting ScFv in healthy and disease biopsies agreed with the macroscopic imaging data. Systemic clearance for ABBV-022-800CW and the non-targeting scFv was found to be similar (Fig. S11).

Expectedly, the systemic clearance for both scFv fusion proteins was slower in primates than in mice. As a response to focal wound injury from TNBS, normal epithelia is replaced by inflammatory cell infiltrate as shown in the H\&E stained tissue section (Fig. 5C, left). In these focal regions, TNC D IHC (Fig. 5C, middle) agreed well with ABBV-022 localization via NIR fluorescence microscopy (Fig. 5C, right).

\section{Discussion}

Increased expression of various ECM components such as fibronectin and tenascin-C in cancer and autoimmune diseases are well-documented $[24,42,43]$. While in vivo targeting of fibronectin extra-domain $A$ is well-characterized for molecular imaging and drug delivery in various autoimmune diseases [19], the previously identified D domain of tenascin $\mathrm{C}$ is largely unexplored for IBD. In addition to sequence alignment showing strong species conservation for TNC D across models, our IHC pointed to unique expression in the lamina propria in clinical IBD colon biopsies and in colonic lesions of the commonly 
used DSS mouse colitis model. Thus, two constructs-an IL-22 immunocytokine fusion (ABBV-022) currently being evaluated in clinical phase I trials and a targeting-only lgG-were evaluated.

Near infrared targeted molecular imaging has played an increasingly important role in preclinical research, ranging from pharmacokinetics to elucidating tissue and cellular level distribution of proteins and low molecular weight molecules $[44,45]$. In this work, the proteins were non-specifically labeled via amine chemistry with IRDye $800 \mathrm{CW}$ to characterize systemic clearance and verify colon specificity in mouse and primate colitis models. In murine colitis, organ level NIR images revealed punctate lesions ranging from the distal to proximal end of the colon. The in vivo saturable binding was confirmed using a high molar blocking dose and this specificity for TNC D was further corroborated by IHC and NIR microscopy. The saturating dose was not identified. As the purpose of this study was to evaluate targeting specificity, a single dose of pharmacologically active, NIR labeled fusion protein was administered $24 \mathrm{~h}$ prior to imaging to minimize potential treatment effects on target expression. Similar to the anti-TNC D IgG-800CW imaging results, a molar excess dose of unlabeled anti-TNC D IgG blocked the NIR signal from ABBV-022-800CW (Fig. S5), suggesting minimal imaging signal contribution from the pharmacologically active IL-22 arm of the fusion protein. Measurements for absolute tenascin expression in preclinical colitis models are rare, though estimates in human ulcerative colitis quantified via Western blotting have been reported [46] and suggest that the fluorescent doses in this study are sub-saturating. With this in mind, we observed higher in vivo signal at the organ and tissue level with the lgG format vs the scFv format. We hypothesize this is due to a combination of faster systemic clearance and reduced ABBV-022 binding affinity for TNC $D$.

The more clinically relevant ABBV-022-800CW was evaluated in a primate colitis model despite moderately lower colon uptake in mouse DSS. While a blocking dose was feasible in murine models, this strategy to assess macroscopic organ specificity was not pursued in the primate colitis model due to challenges in formulation. In the primate ex vivo images, although the MFI for diseased tissues was 2fold higher than healthy tissue, we were concerned about non-specific imaging signal due to inflammation [25]. To best estimate this, analogous imaging data were collected for a non-targeting IL-22 ScFv fusion protein with similar pharmacokinetic properties and molecular weight. In combination with cellular level microscopy, the data suggested high non-specific NIR signal in the macroscopic colon NIR scans accompanying a more modest targeting profile for ABBV-022-800 CW confirmed through microscopy and IHC. These data highlight the important interplay between imaging agent binding affinity and in vivo target expression. In the preclinical rodent models, we hypothesize the estimated TNC D expression in colonic lesions was high enough to mitigate the impacts of the reduced affinity for the scFv fusion protein.

Empirically, extracellular receptors have some of the highest contrast values for imaging agents, and delivery pathways of therapeutic proteins-such as ADCs-share similarities with imaging agent delivery. However, a distinction for IBD is immunological targets are often soluble or surface expressed in subsets of motile cells $[47,48]$. Therefore, targeted IBD therapeutics of local cytokine delivery should avoid internalization pathways for the targeting ligand. This makes ECM targets attractive, where a high local 
ECM concentration drives preferential accumulation in disease shortly following dosing, accompanied by a gradual dissociation post-washout to drive efficacy. Unlike cell surface expressed receptors, where probe design steps can be taken to improve internalization and residualization of the targeting ligand to mitigate poor in vivo expression, imaging ECM targets largely relies on engineering stronger affinity to the target and faster blood clearance for efficient delivery and rapid washout. However, screening for high affinity binders risk non-specific binding, and altering blood clearance may prove challenging without significant changes in protein format. Thus, a practical alternative is to identify ECM targets with high absolute local expression unique to disease tissue. The murine blocking experiments estimate absolute expression of TNC D at the site of lesions is likely higher than $100 \mathrm{nM}$ with a conservative estimate of lesion volume fraction in the colon through whole organ digest (Fig. S4). This estimate is similar to cell surface molecular imaging targets present in autoimmune diseases such as type 1 diabetes and various cancers with high reported imaging target to background ratio (TBR) [49-51], although further measurements are recommended.

While beyond the scope of this work, several possible challenges exist for delivery-based applications targeting TNC D. First, as a diagnostic target, NIR imaging faces depth limitations and a radiolabeled probe will be necessary for clinical translation. Depending on the size and distribution of human lesions, accurate in vivo quantification can prove challenging. Accurately quantifying tracer uptake in smaller lesions may face similar challenges as other autoimmune targets (i.e., beta cell mass quantification for type 1 diabetes). Second, ideal imaging agent properties and ideal drug delivery ligand properties differ. For uniquely imaging applications, TBR can be improved by engineering affinity to slow dissociation relative to washout. While this may increase total protein concentration in diseased tissue for a drugdelivery application, ECM dissociation may be necessary to drive pharmacology in scenarios where the immunological target might be soluble or expressed in nearby tissue. The quantitative impacts of these mechanisms are difficult to estimate through experiments alone; here, a mechanistic and predictive framework can aid successful drug design. In conclusion, our data showcase protein imaging agents with strong specificity for a highly expressed colon ECM unique to disease.

\section{Declarations}

\section{Competing Interests}

L.Z., C.S.P., V.Z.S., C.M.N., J.E.E., H.L.K., S.B., G.R.L., B.L.M., A.J.S., and S.M. are current employees of AbbVie. K.T.H., and S.M.G. were employees of AbbVie at the time of the study. The design, study conduct, and financial support for the experiments were provided by AbbVie. AbbVie participated in the interpretation of data, review, and approval of the publication. The authors wish to thank Dr. Jochen Salfeld and Dr. Trudi Veldman for scientific input, suggestions, and proofreading the manuscript.

\section{Data Availability}

The datasets generated during and/or analyzed during the current study are available from the corresponding author on reasonable request. 


\section{References}

1. Lautenschläger C, Schmidt C, Fischer D, Stallmach A. Drug delivery strategies in the therapy of inflammatory bowel disease. Adv Drug Deliv Rev. 2014;71:58-76.

2. Colombel J-F, D'haens G, Lee W-J, Petersson J, Panaccione R. Outcomes and Strategies to Support a Treat-to-target Approach in Inflammatory Bowel Disease: A Systematic Review. Journal of Crohn's Colitis. 2020;14:254-66.

3. Palmela C, Torres J, Cravo M. New Trends in Inflammatory Bowel Disease. GE Portuguese Journal of Gastroenterology. 2015;22:103-11.

4. Neurath MF. Cytokines in inflammatory bowel disease. Nat Rev Immunol. 2014;14:329-42.

5. Belali N, Wathoni N, Muchtaridi M. Advances in orally targeted drug delivery to colon. J Adv Pharm Technol Res. 2019;10:100-6.

6. Schreiber S, Fedorak RN, Nielsen OH, Wild G, Williams CN, Nikolaus S, et al. Safety and efficacy of recombinant human interleukin 10 in chronic active Crohn's disease. Gastroenterology. 2000;119:1461-72.

7. Hanson ML, Hixon JA, Li W, Felber BK, Anver MR, Stewart CA, et al. Oral Delivery of IL-27 Recombinant Bacteria Attenuates Immune Colitis in Mice. Gastroenterology. 2014;146:210-21.e13.

8. Tang K-Y, Lickliter J, Huang Z-H, Xian Z-S, Chen H-Y, Huang C, et al. Safety, pharmacokinetics, and biomarkers of F-652, a recombinant human interleukin-22 dimer, in healthy subjects. Cellular Molecular Immunology Nature Publishing Group. 2019;16:473-82.

9. Doll F, Schwager K, Hemmerle T, Neri D. Murine analogues of etanercept and of F8-IL10 inhibit the progression of collagen-induced arthritis in the mouse. Arthritis Res Ther. 2013;15:R138.

10. Villa A, Trachsel E, Kaspar M, Schliemann C, Sommavilla R, Rybak J-N, et al. A high-affinity human monoclonal antibody specific to the alternatively spliced EDA domain of fibronectin efficiently targets tumor neo-vasculature in vivo. Int J Cancer. 2008;122:2405-13.

11. Schwager K, Kaspar M, Bootz F, Marcolongo R, Paresce E, Neri D, et al. Preclinical characterization of DEKAVIL (F8-IL10), a novel clinical-stage immunocytokine which inhibits the progression of collageninduced arthritis. Arthritis Res Ther. 2009;11:R142.

12. Bootz F, Ziffels B, Neri D. Antibody-Based Targeted Delivery of Interleukin-22 Promotes Rapid Clinical Recovery in Mice With DSS-Induced Colitis. Inflamm Bowel Dis. 2016;22:2098-105.

13. Petrey AC, de la Motte CA. The extracellular matrix in IBD: a dynamic mediator of inflammation. Curr Opin Gastroenterol. 2017;33:234-8.

14. Riedl S, Tandara A, Reinshagen M, Hinz U, Faissner A, Bodenmüller $H$, et al. Serum tenascin-C is an indicator of inflammatory bowel disease activity. Int J Colorectal Dis. 2001;16:285-91.

15. Reardon DA, Akabani G, Coleman RE, Friedman AH, Friedman HS, Herndon JE, et al. Phase II trial of murine (131)I-labeled antitenascin monoclonal antibody 81C6 administered into surgically created resection cavities of patients with newly diagnosed malignant gliomas. J Clin Oncol. 2002;20:138997. 
16. Riva P, Franceschi G, Frattarelli M, Riva N, Guiducci G, Cremonini AM, et al. 131I radioconjugated antibodies for the locoregional radioimmunotherapy of high-grade malignant glioma--phase I and II study. Acta Oncol. 1999;38:351-9.

17. Rizzieri DA, Akabani G, Zalutsky MR, Coleman RE, Metzler SD, Bowsher JE, et al. Phase 1 trial study of 131/-labeled chimeric $81 \mathrm{C6}$ monoclonal antibody for the treatment of patients with non-Hodgkin lymphoma. Blood. 2004;104:642-8.

18. Brack SS, Silacci M, Birchler M, Neri D. Tumor-Targeting Properties of Novel Antibodies Specific to the Large Isoform of Tenascin-C. Clin Cancer Res. 2006;12:3200-8.

19. Bruijnen STG, Chandrupatla DMSH, Giovanonni L, Neri D, Vugts DJ, Huisman MC, et al. F8-IL10: A New Potential Antirheumatic Drug Evaluated by a PET-Guided Translational Approach. Mol Pharm. 2019;16:273-81.

20. Islam MS, Kusakabe M, Horiguchi K, lino S, Nakamura T, Iwanaga K, et al. PDGF and TGF- $\beta$ promote tenascin-C expression in subepithelial myofibroblasts and contribute to intestinal mucosal protection in mice. Br J Pharmacol. 2014;171:375-88.

21. Kawamura T, Yamamoto M, Suzuki K, Suzuki Y, Kamishima M, Sakata M, et al. Tenascin-C Produced by Intestinal Myofibroblasts Promotes Colitis-associated Cancer Development Through Angiogenesis. Inflamm Bowel Dis. 2019;25:732-41.

22. Rybak J-N, Ettorre A, Kaissling B, Giavazzi R, Neri D, Elia G. In vivo protein biotinylation for identification of organ-specific antigens accessible from the vasculature. Nat Methods. 2005;2:2918.

23. Conrotto P, Roesli C, Rybak J, Kischel P, Waltregny D, Neri D, et al. Identification of new accessible tumor antigens in human colon cancer by ex vivo protein biotinylation and comparative mass spectrometry analysis. Int J Cancer. 2008;123:2856-64.

24. Bootz F, Neri D. Immunocytokines: a novel class of products for the treatment of chronic inflammation and autoimmune conditions. Drug Discovery Today. 2016;21:180-9.

25. Zhang L, Bhatnagar S, Deschenes E, Thurber GM. Mechanistic and quantitative insight into cell surface targeted molecular imaging agent design. Scientific Reports [Internet]. 2016 [cited 2021 Apr 6];6. Available from: http://www.nature.com/articles/srep25424.

26. Tolmachev V, Orlova A. Affibody Molecules as Targeting Vectors for PET Imaging. Cancers (Basel). 2020;12.

27. Willmann JK, van Bruggen N, Dinkelborg LM, Gambhir SS. Molecular imaging in drug development. Nat Rev Drug Discovery. 2008;7:591-607.

28. Ongaro T, Gouyou B, Stringhini M, Corbellari R, Neri D, Villa A. A novel format for recombinant antibody-interleukin-2 fusion proteins exhibits superior tumor-targeting properties in vivo. Oncotarget. 2020;11:3698-711.

29. Zegers CML, Rekers NH, Quaden DHF, Lieuwes NG, Yaromina A, Germeraad WTV, et al. Radiotherapy Combined with the Immunocytokine L19-IL2 Provides Long-lasting Antitumor Effects. Clin Cancer Res. 2015;21:1151-60. 
30. Polakis P. Antibody Drug Conjugates for Cancer Therapy. Esbenshade TA, editor. Pharmacological Reviews. 2016;68:3-19.

31. Diamantis N, Banerji U. Antibody-drug conjugates-an emerging class of cancer treatment. British Journal of Cancer Nature Publishing Group. 2016;114:362-7.

32. Zhang S, Langer R, Traverso G. Nanoparticulate drug delivery systems targeting inflammation for treatment of inflammatory bowel disease. Nano Today. 2017;16:82-96.

33. Freise AC, Zettlitz KA, Salazar FB, Tavaré R, Tsai W-TK, Chatziioannou AF, et al. Immuno-PET in Inflammatory Bowel Disease: Imaging CD4-Positive T Cells in a Murine Model of Colitis. J Nucl Med. 2018;59:980-5.

34. Higashikawa K, Akada N, Yagi K, Watanabe K, Kamino S, Kanayama Y, et al. Exploration of target molecules for molecular imaging of inflammatory bowel disease. Biochem Biophys Res Commun. 2011;410:416-21.

35. Bettenworth D, Reuter S, Hermann S, Weckesser M, Kerstiens L, Stratis A, et al. Translational 18F-FDG PET/CT Imaging to Monitor Lesion Activity in Intestinal Inflammation. Journal of Nuclear Medicine Society of Nuclear Medicine. 2013;54:748-55.

36. Treglia G, Quartuccio N, Sadeghi R, Farchione A, Caldarella C, Bertagna F, et al. Diagnostic performance of Fluorine-18-Fluorodeoxyglucose positron emission tomography in patients with chronic inflammatory bowel disease: A systematic review and a meta-analysis. Journal of Crohn's Colitis. 2013;7:345-54.

37. Cilliers C, Nessler I, Christodolu N, Thurber GM. Tracking Antibody Distribution with Near-Infrared Fluorescent Dyes: Impact of Dye Structure and Degree of Labeling on Plasma Clearance. Mol Pharmaceutics American Chemical Society. 2017;14:1623-33.

38. Oliveira S, Cohen R, Walsum MS, van Dongen GA, Elias SG, van Diest PJ, et al. A novel method to quantify IRDye800CW fluorescent antibody probes ex vivo in tissue distribution studies. EJNMMI Res. 2012;2:50.

39. Cilliers C, Liao J, Atangcho L, Thurber GM. Residualization Rates of Near-Infrared Dyes for the Rational Design of Molecular Imaging Agents. Mol Imaging Biol. 2015;17:757-62.

40. de Jongh SJ, Vrouwe JPM, Voskuil FJ, Schmidt I, Westerhof J, Koornstra JJ, et al. The Optimal Imaging Window for Dysplastic Colorectal Polyp Detection Using c-Met-Targeted Fluorescence Molecular Endoscopy. J Nucl Med. 2020;61:1435-41.

41. Burggraaf J, Kamerling IMC, Gordon PB, Schrier L, de Kam ML, Kales AJ, et al. Detection of colorectal polyps in humans using an intravenously administered fluorescent peptide targeted against c-Met. Nature Medicine Nature Publishing Group. 2015;21:955-61.

42. Borgia B, Roesli C, Fugmann T, Schliemann C, Cesca M, Neri D, et al. A Proteomic Approach for the Identification of Vascular Markers of Liver Metastasis. Can Res. 2010;70:309-18.

43. Rybak J-N, Roesli C, Kaspar M, Villa A, Neri D. The Extra-domain A of Fibronectin Is a Vascular Marker of Solid Tumors and Metastases. Can Res. 2007;67:10948-57. 
44. Hilderbrand SA, Weissleder R. Near-infrared fluorescence: application to in vivo molecular imaging. Curr Opin Chem Biol. 2010;14:71-9.

45. Bhatnagar S, Deschenes E, Liao J, Cilliers C, Thurber GM. Multichannel Imaging to Quantify Four Classes of Pharmacokinetic Distribution in Tumors. J Pharm Sci. 2014;103:3276-86.

46. Dueck M, Riedl S, Hinz U, Tandara A, Möller P, Herfarth $C$, et al. Detection of tenascin-C isoforms in colorectal mucosa, ulcerative colitis, carcinomas and liver metastases. Int J Cancer. 1999;82:47783.

47. Neurath MF. Current and emerging therapeutic targets for IBD. Nature Reviews Gastroenterology Hepatology Nature Publishing Group. 2017;14:269-78.

48. Monteleone G, Pallone F, MacDonald TT. Emerging immunological targets in inflammatory bowel disease. Curr Opin Pharmacol. 2011;11:640-5.

49. Zhang L, Thurber GM. Quantitative Impact of Plasma Clearance and Down-regulation on GLP-1 Receptor Molecular Imaging. Mol Imaging Biol. 2016;18:79-89.

50. Wittrup KD, Thurber GM, Schmidt MM, Rhoden JJ. Chapter ten - Practical Theoretic Guidance for the Design of Tumor-Targeting Agents. In: Wittrup KD, Verdine GL, editors. Methods in Enzymology [Internet]. Academic Press; 2012 [cited 2021 Apr 6]. p. 255-68. Available from: https://www.sciencedirect.com/science/article/pii/B9780123969620000100.

51. Schmidt MM, Wittrup KD. A modeling analysis of the effects of molecular size and binding affinity on tumor targeting. Mol Cancer Ther American Association for Cancer Research. 2009;8:2861-71.

\section{Tables}

\section{Table 1}

\begin{tabular}{lclc} 
Reagents & $\mathbf{k}_{\text {on }}\left(\mathbf{M}^{-1} \mathbf{s}^{-1}\right)$ & $\mathbf{k}_{\text {off }}\left(\mathbf{s}^{-1}\right)$ & $\mathbf{K}_{\mathbf{D}}(\mathbf{M})$ \\
\hline ABBV-022 & $5.5 \times 10^{4}$ & $7.5 \times 10^{-2}$ & $1.4 \times 10^{-6}$ \\
ABBV-022-800CW & $5.5 \times 10^{4}$ & $7.6 \times 10^{-2}$ & $1.4 \times 10^{-6}$ \\
Anti-TNC D lgG & $1.4 \times 10^{5}$ & $7.1 \times 10^{-2}$ & $5.0 \times 10^{-7}$ \\
Anti-TNC D lgG-800CW & $1.3 \times 10^{5}$ & $7.1 \times 10^{-2}$ & $5.3 \times 10^{-7}$
\end{tabular}


Table 2

\begin{tabular}{lcccc} 
Group & $\mathrm{C}_{0}(\mathbf{n M})$ & Fraction fast & $\mathbf{t}_{1 / 2, \alpha}(\mathbf{h})$ & $\mathbf{t}_{1 / 2, \beta}(\mathbf{h})$ \\
\hline Anti-TNC D IgG-800CW & $440 \pm 30$ & $0.5 \pm 0.1$ & $3 \pm 2$ & $19 \pm 3$ \\
Anti-TNC D IgG-800CW + blocking & $530 \pm 40$ & $0.6 \pm 0.1$ & $1.7 \pm 0.6$ & $21 \pm 3$ \\
ABBV-022-800CW & $570 \pm 80$ & $0.6 \pm 0.1$ & $0.5 \pm 0.2$ & $5.2 \pm 0.3$ \\
Isotype IgG-800CW & $420 \pm 40$ & $0.5 \pm 0.1$ & $0.3 \pm 0.1$ & $20 \pm 1$
\end{tabular}

Figures

Figure 1

A
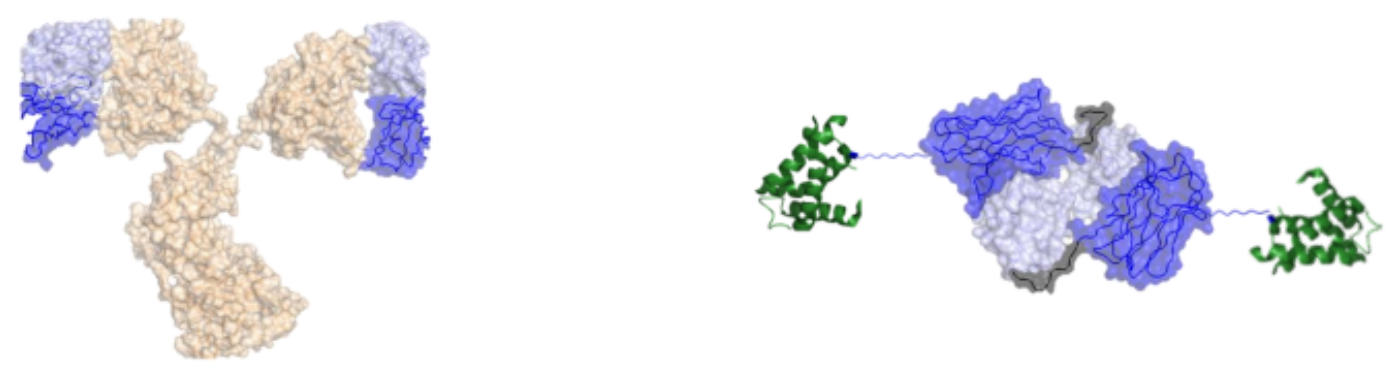

B

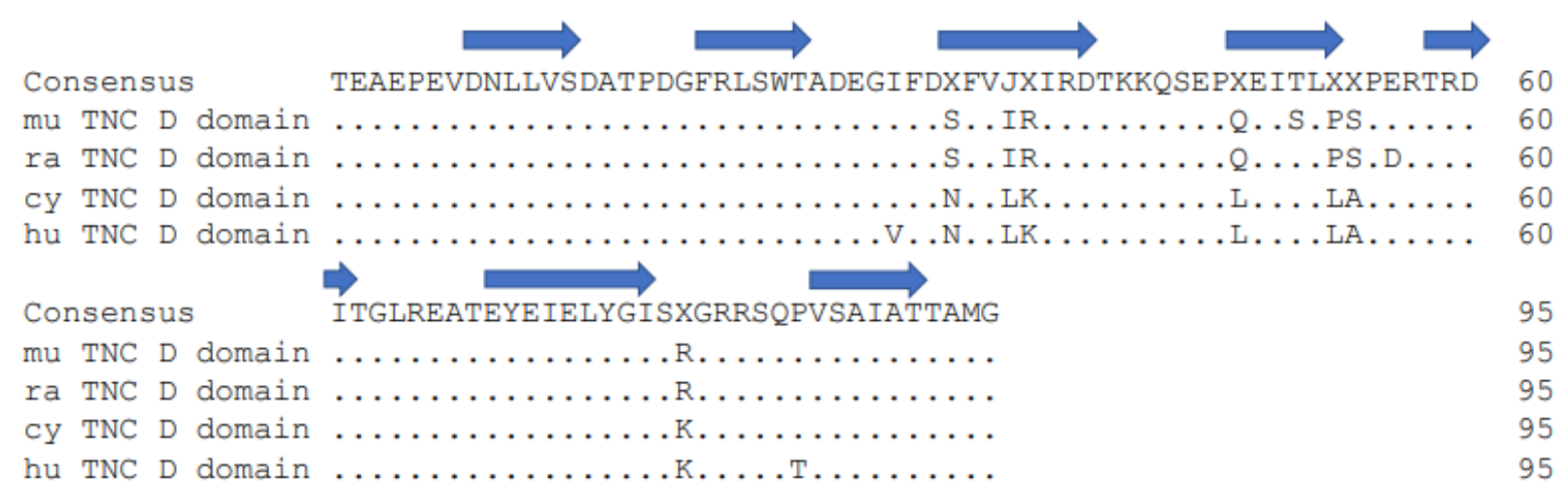

Figure 1 
(A) Surface representation of TNC D IgG (wheat) with variable heavy (dark blue) and variable light (light blue) domains. Variable heavy and variable light domains from the IgG were translated into an ScFv using a short, flexible glycine-serine linker (black). IL-22 shown in green (PDB ID: 3DLQ) connected to the scFv via a flexible (G4S)3 linker. (B) Sequence alignment for TNC D domains from mouse, rat, cynomolgus monkey, and human. Domain annotations derived from available tenascin C FNIII structure (PDB ID: 1TEN).

\section{Figure 2}
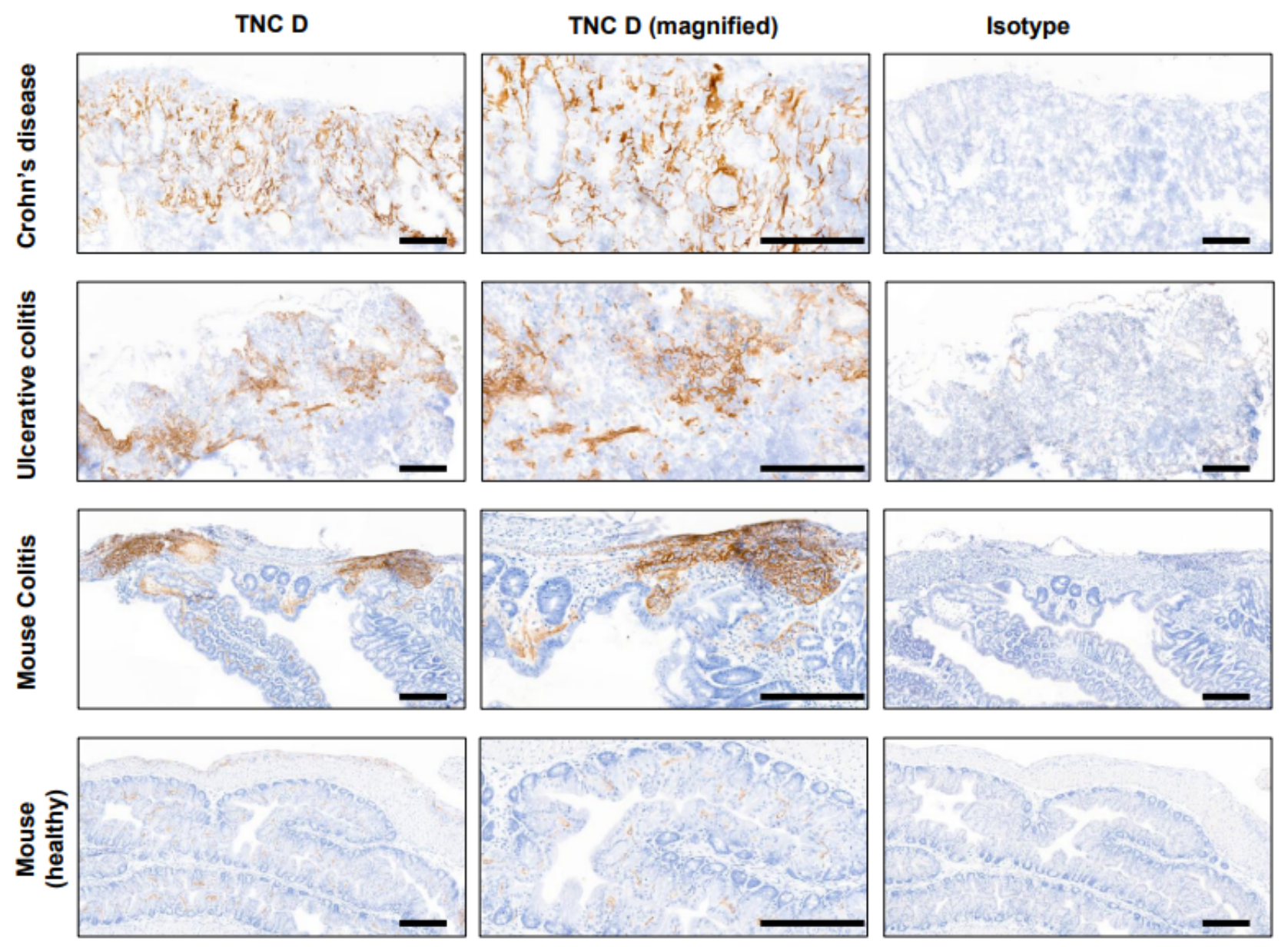

\section{Figure 2}

Immunohistochemistry panel for TNC D in colon. Target validation in sections of human and animal colon tissue with antibodies specific to the alternatively spliced D domain of tenascin C. Scale bars, 200 $\mu \mathrm{m}$. 


\section{Figure 3}

A

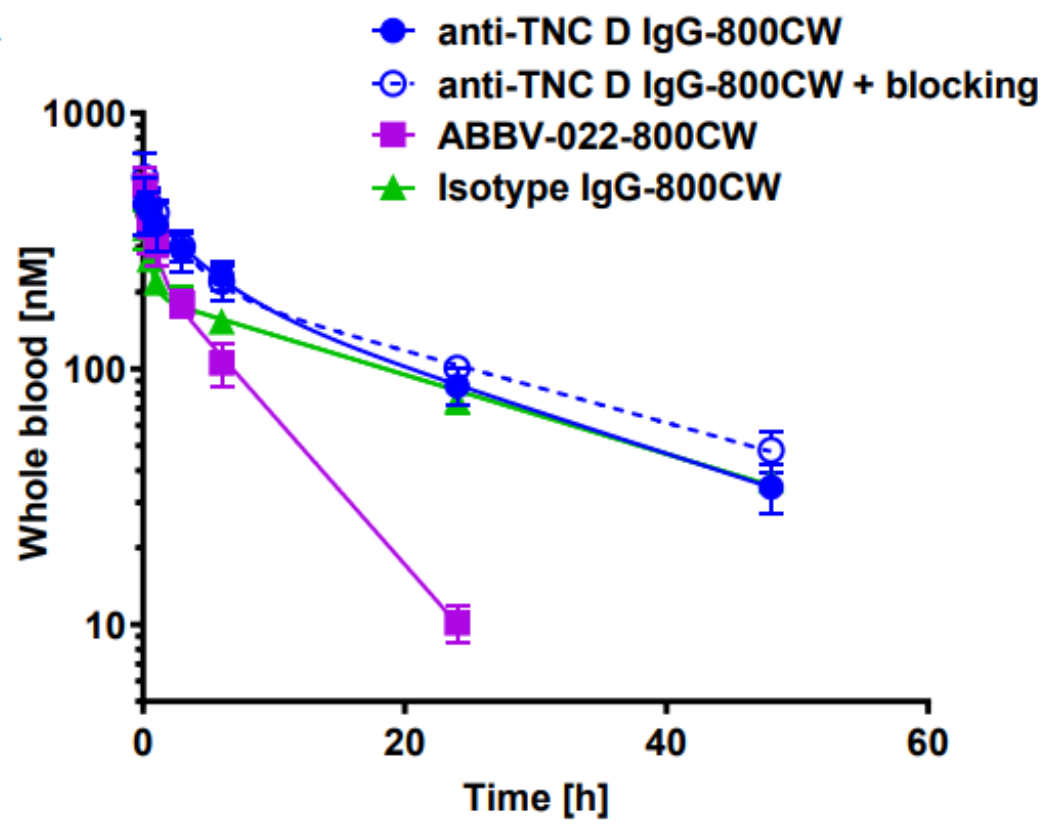

B
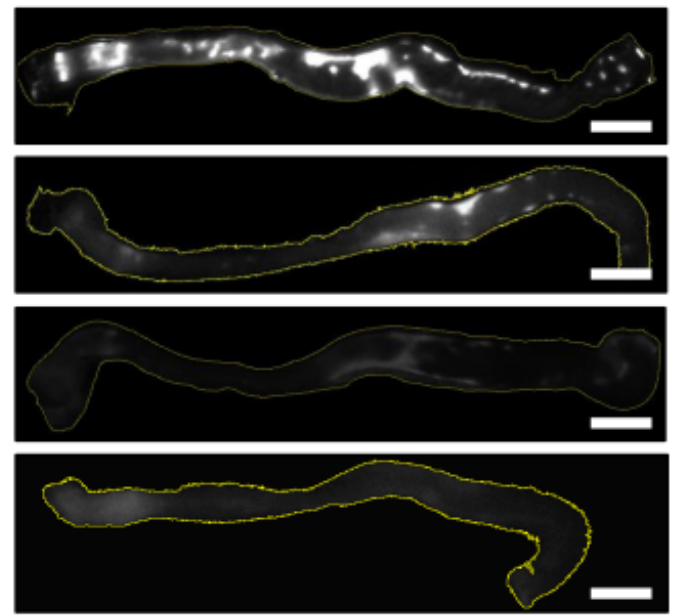

Figure 3

(A) Blood clearance is faster for ABBV-022-800CW compared to lgG formats. Addition of an anti-TNC D IgG blocking dose does not significantly impact the near infrared dose. Isotype IgG-800CW exhibits a similar systemic clearance profile compared to the anti-TNC D IgG-800CW. (B) From top to bottom: antiTNC D lgG-800CW, ABBV-022-800CW, anti-TNC D-800CW + blocking; isotype IgG-800CW. Macroscopic organ images show punctate spots at the site of lesions for anti-TNC D IgG-800CW and ABBV-022800CW groups. Reduced lesion-associated signal observed in the blocking and isotype lgG groups. Scale bars, $5 \mathrm{~mm}$. 


\section{Figure 4}

A

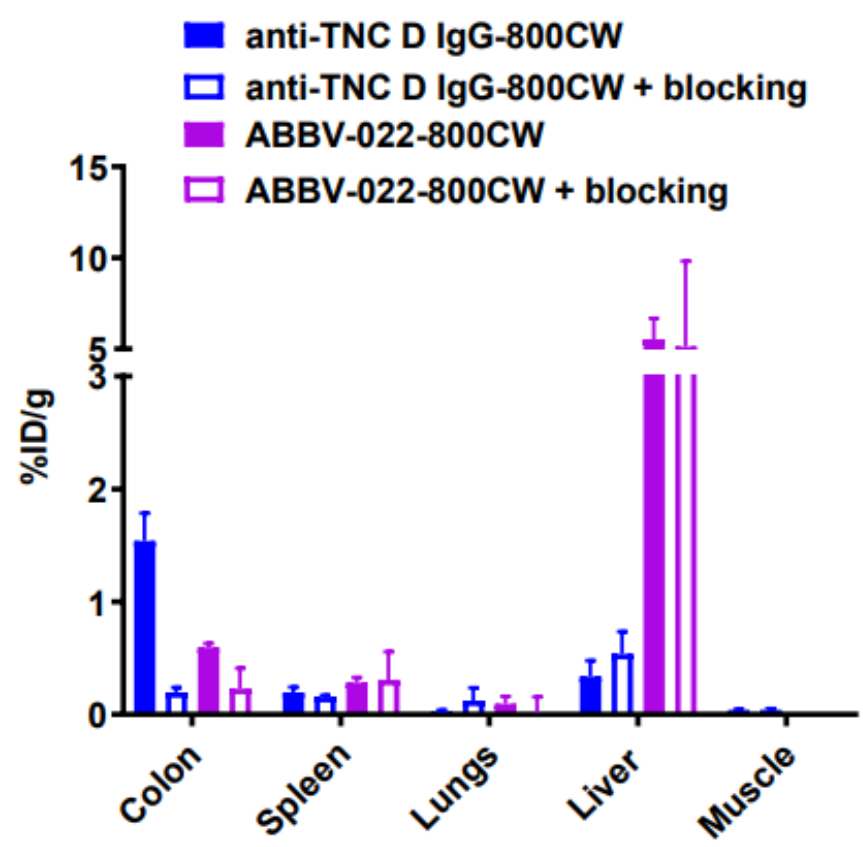

B
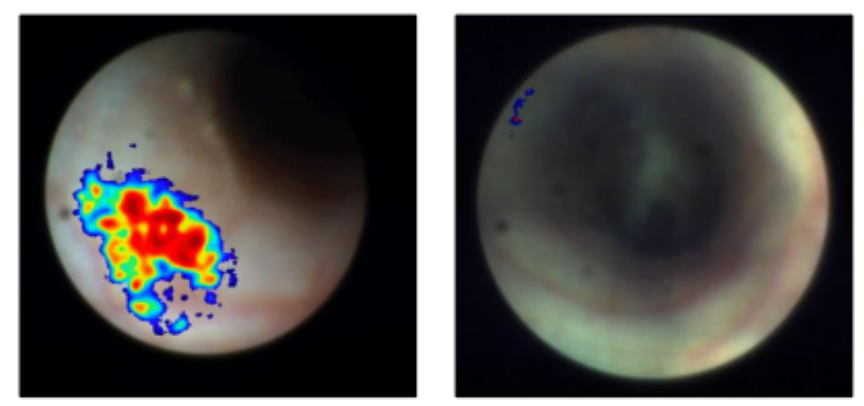

C
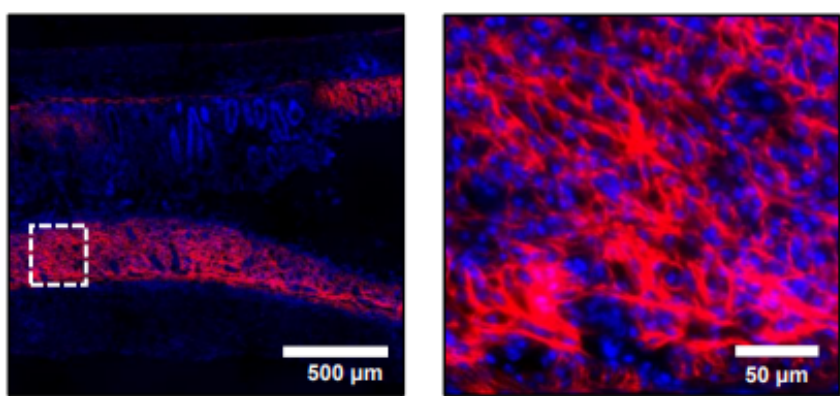

Figure 4

(A) Ex vivo biodistribution data show a reduction in disease colon signal with the addition of TNC D blocking dose. No differences in uptake are observed in non-target organs. Higher non-specific uptake is observed in the liver. (B) Near infrared molecular endoscopy frame capture for dosed (left) and un-dosed (right) animals in mouse DSS colitis. A colonic lesion is visible for the animal dosed with ABBV-022800CW. (C) Cellular level distribution for anti-TNC D IgG-800CW. Cell nuclei are stained with Hoechst 33342 (blue); NIR channel (red). 


\section{Figure 5}

A

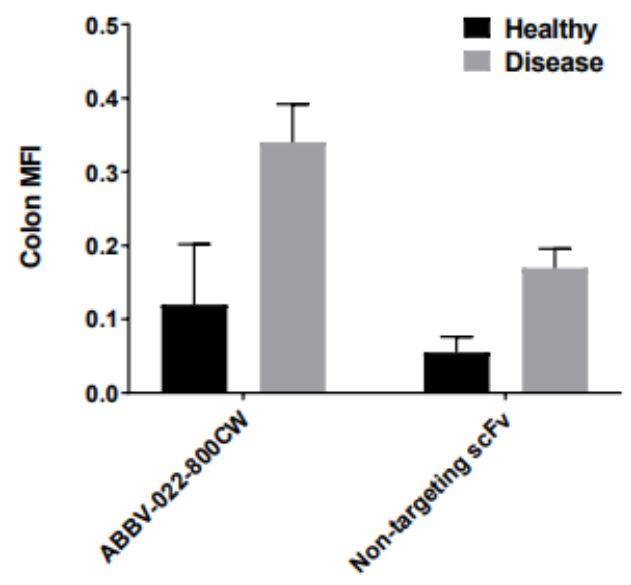

B

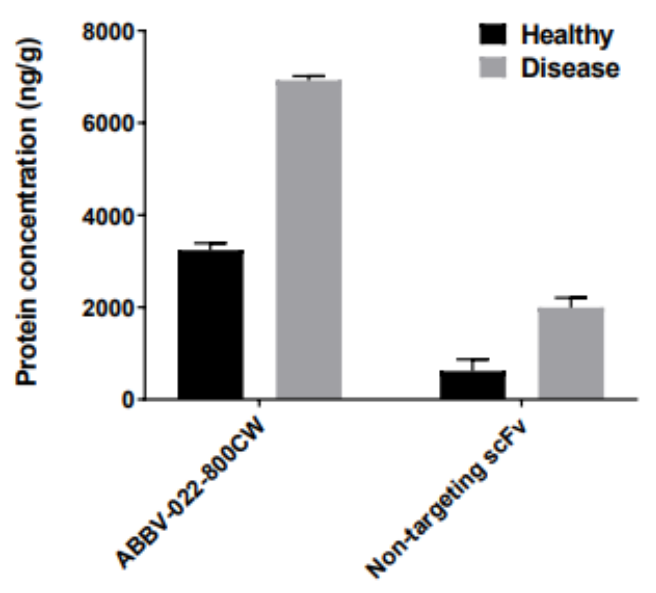

C
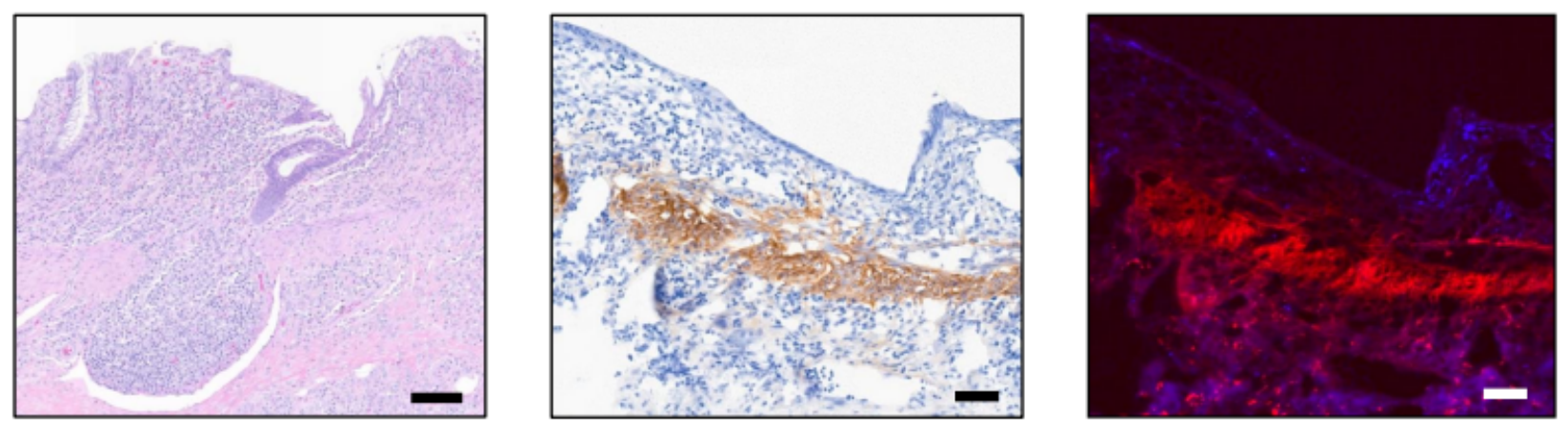

\section{Figure 5}

(A) Colon segment mean fluorescent intensity plots for cynomolgus monkeys dosed with either ABBV$022-800 \mathrm{CW}$ or a non-targeting scFv. Segments of diseased and healthy colon were identified by eye from the same animal for each group. Increased signal observed in disease vs healthy tissue for both proteins (B) Biopsy homogenates show a higher concentration of dosed NIR fluorescent protein in diseased vs healthy tissue for both proteins. (C) From left to right: diseased cynomolgus colon H\&E showing immune cell infiltrate, TNC D IHC in diseased colon, serial section NIR image in agreement with IHC. Scale bars, $100 \mu \mathrm{m}$.

\section{Supplementary Files}

This is a list of supplementary files associated with this preprint. Click to download.

- 72767SupplementaryInformationv2.docx 